\title{
On the excitation of trapped and leaky modes in coronal slabs
}

\author{
J. Terradas, R. Oliver, and J. L. Ballester
}

\author{
Departament de Física, Universitat de les Illes Balears, 07122 Palma de Mallorca, Spain \\ e-mail: [jaume.terradas; ramon.oliver; dfsjlb@]@uib.es
}

Received 5 April 2005 / Accepted 23 June 2005

\begin{abstract}
We study the excitation of fast waves in solar coronal loops modeled as smoothed, dense plasma slabs in a uniform magnetic field. We analyse the features of the trapped and leaky modes which result from an initial disturbance generated in the loop. By solving the time-dependent problem we find that the stationary state of the slab motion is described by the trapped normal modes and that the transient between the initial and the stationary phase is dominated by wave leakage. The period and duration of this transient (what we call the impulsive leaky phase) are in perfect agreement with the corresponding values of leaky modes calculated from the normal mode analysis.
\end{abstract}

Key words. Sun: corona - Sun: magnetic fields - waves

\section{Introduction}

Transversal coronal loop oscillations have been widely reported in recent years using Transition Region and Coronal Explorer (TRACE) observations. These kind of oscillations have been interpreted as standing kink fast magnetohydrodynamic (MHD) waves (Aschwanden et al. 1999, 2002; Nakariakov et al. 1999; Nakariakov \& Ofman 2001; Schrijver et al. 2002) since they produce displacements of the loop axis and the loop footpoints seem to be anchored in the solar photosphere.

The theory of MHD waves in loops has been developed in detail for different geometries using normal mode analysis. Loops modeled as straight magnetic slabs of enhanced density have been studied in detail by Edwin \& Roberts (1982), while waves in loops modeled as straight cylindrical flux tubes have been analysed by Spruit (1982); Edwin \& Roberts (1983); Cally (1986, 2003); Díaz et al. (2004). The spectrum of normal modes is quite rich and is basically formed by two classes of modes: trapped modes, with real frequencies, and leaky modes, with complex frequencies. Strictly speaking, leaky modes (also termed pseudo-modes or quasinormal modes) are not normal modes since their amplitude in the coronal medium increases with the distance from the loop. The features of leaky waves in a cylinder have been analysed in great detail by Cally (1986, 2003), who has classified them and derived analytical expressions for their period and damping time. An appealing feature of these modes is that they represent propagating waves from the density enhancement. As a consequence, an oscillating loop can lose some energy into the surrounding by means of the excitation of one or more leaky modes. Thus, these modes are likely to be quite relevant from the energy point of view.

Although the features of trapped and leaky modes are well known, their role concerning the initial-value problem has not been investigated in much detail. The time-dependent problem of the excitation of loop oscillations has been analysed in several works. These studies have focused on the excitation of waves in unbounded slabs (Rae \& Roberts 1982; Roberts et al. 1984; Murawski \& Roberts 1993a,b,c; Huang 1996; Huang et al. 1999; Musielak et al. 2000; Selwa \& Murawski 2004b) or unbounded cylindrical loops (Selwa \& Murawski 2004a), allowing perturbations to propagate along and across the loop. Only a few works have solved the time-dependent problem in line-tied loops (Uralov 2003; Terradas \& Ofman 2004; Terradas et al. 2005).

Here we consider a line-tied slab model and solve the timedependent problem of the excitation of coronal loop oscillations. In general, an initial disturbance is likely to deposit some energy in the trapped modes, while some energy will be emitted via the leaky waves. We concentrate on the conditions that lead to the excitation of trapped modes and, specially, leaky modes and compare the results of the time-dependent simulations with the information provided by the normal (trapped and leaky) modes.

This paper is organised as follows. In Sect. 2 the loop model and the basic MHD equations describing fast waves are presented. In Sect. 3 the features of trapped and leaky modes are reviewed and estimations of the real and imaginary part of the frequency of leaky modes are derived. In Sect. 4 the time-dependent problem is considered and the resulting velocity profiles are studied for several initial perturbations. Finally in Sect. 5 the main conclusions are drawn.

\section{Loop model and basic equations}

The equilibrium configuration used to model a coronal loop of radius $a$ consists of a homogeneous straight slab described in 
Cartesian coordinates. We assume the following plasma density profile

$\rho= \begin{cases}\rho_{\mathrm{i}}, & \text { if }|x| \leq a \\ \rho_{\mathrm{e}}, & \text { if }|x|>a\end{cases}$

where $\rho_{\mathrm{i}}$ is the density inside the slab and $\rho_{\mathrm{e}}$ denotes the gas density in the loop environment (since coronal loops are regions of gas density enhancement $\rho_{\mathrm{i}}>\rho_{\mathrm{e}}$ ). Moreover, the slab is permeated by a vertical and uniform magnetic field $\left(\boldsymbol{B}=B_{0} \boldsymbol{e}_{z}\right)$. The Alfvén speed, $v_{\mathrm{A}}=B_{0} / \sqrt{\mu \rho}$, takes the value $v_{\mathrm{Ai}}$ inside the slab and $v_{\mathrm{Ae}}$ in the surrounding coronal medium.

Linear perturbations about this equilibrium can be readily described using the equations of ideal MHD. In the zero- $\beta$ limit, valid under coronal conditions, they reduce to the wellknown wave equation for the fast modes,

$$
\frac{\partial^{2} v_{x}}{\partial t^{2}}=v_{\mathrm{A}}^{2}\left(\frac{\partial^{2} v_{x}}{\partial x^{2}}+\frac{\partial^{2} v_{x}}{\partial z^{2}}\right),
$$

where $v_{x}$ is the velocity component transversal to the unperturbed magnetic field. Since $\beta=0$ slow modes are absent $\left(v_{z}=0\right)$.

We Fourier analyse in the $z$-direction, i.e. we assume that the perturbations are of the form $\mathrm{e}^{-\mathrm{i} k_{z} z}$. Hence, Eq. (2) reduces to the Klein-Gordon equation,

$$
\frac{\partial^{2} v_{x}}{\partial t^{2}}=v_{\mathrm{A}}^{2} \frac{\partial^{2} v_{x}}{\partial x^{2}}-\omega_{\mathrm{c}}^{2} v_{x}
$$

where $\omega_{\mathrm{c}}=v_{\mathrm{A}} k_{z}$. Thus, after introducing the longitudinal wavenumber $k_{z}$ the two-dimensional wave equation reduces to a one-dimensional equation, and by selecting the appropriate value of this parameter the effect of line-tying can be easily incorporated. For the fundamental kink mode, which is the mode that seems to be observed, the loop has a node at each footpoint and thus $k_{z}=2 \pi / 2 L, L$ being the length of the loop. Note that the time a wave needs to travel along the entire loop to feel the effect of the photosphere is neglected, and in our model the loop instantly feels the effect of line-tying.

For a uniform Alfvén speed $\left(v_{\mathrm{Ai}}=v_{\mathrm{Ae}}\right)$, the solution of the time-dependent problem under some initial perturbations has been analysed by Rae \& Roberts (1982) and more recently by Terradas et al. (2005). Wave propagation is dispersive, with short wavelengths propagating faster than longer wavelengths. The time-dependent solution is characterised by a wavefront travelling at the Alfvén speed and by a wake behind this pulse oscillating at the frequency $\omega_{\mathrm{c}}$. However, for the loop model $v_{\mathrm{Ai}}<v_{\mathrm{Ae}}$ and the Alfvén velocity changes in the $x$-direction. As a consequence, the analytical solution of the initial value problem, involving temporal Laplace transforms, is much more complex. For this reason we have solved Eq. (3) numerically using standard techniques.

\section{Normal modes}

Before considering the time-dependent problem, we briefly describe the main oscillatory features of our slab model, given by the normal mode analysis. For such kind of analysis, a time dependence of the form $\mathrm{e}^{\mathrm{i} \omega t}$ is prescribed in Eq. (3) and then an eigenvalue problem has to be solved (see Edwin \& Roberts 1982). The corresponding dispersion relations are

$\tanh k_{\mathrm{i}} a=-\frac{k_{\mathrm{e}}}{k_{\mathrm{i}}}$

for the kink modes (even parity about $x=0$ ) and

$\operatorname{coth} k_{\mathrm{i}} a=-\frac{k_{\mathrm{e}}}{k_{\mathrm{i}}}$

for the sausage modes (odd parity about $x=0$ ), where

$k_{\mathrm{e}}^{2}=k_{z}^{2}-\frac{\omega^{2}}{v_{\mathrm{Ae}}^{2}}$,

and

$k_{\mathrm{i}}^{2}=k_{z}^{2}-\frac{\omega^{2}}{v_{\mathrm{Ai}}^{2}}$.

These transcendental dispersion relations have essentially two types of solutions. The first kind of modes have real frequency and correspond to trapped waves, whereas the second ones have complex frequency and represent leaky waves. In Fig. 1a we have plotted the real part of the frequency for both families of modes (note that only the fundamental kink and sausage modes and the first kink harmonic are displayed) as a function of $a / L$ under coronal loop conditions $\left(\rho_{\mathrm{i}} / \rho_{\mathrm{e}}=3\right.$ according to Aschwanden et al. 2003). Since we are in the situation $v_{\mathrm{Ai}}<v_{\mathrm{Ae}}$, only body waves are present in the dispersion diagram and surface waves are not permitted (see Edwin \& Roberts 1982). The main properties of trapped and leaky modes are presented next.

\subsection{Trapped modes}

Modes with real frequency below the frequency $\omega_{\mathrm{c}}=k_{z} v_{\mathrm{Ae}}$ (represented by a dotted line in Fig. 1a) are trapped; these solutions correspond to standing oscillations of the slab. For $a / L \ll 1$, such as in typical coronal loops, only the fundamental kink mode is trapped (with a frequency that tends to $\omega_{\mathrm{c}}$ as $a / L \rightarrow 0$ ), while all the other modes are trapped only if $a / L$ is larger than some critical value. These critical values are located in the dispersion diagram where the frequency of a trapped mode intersects with $\omega_{\mathrm{c}}$, and are given by

$(a / L)_{\mathrm{c}}=\frac{v_{\mathrm{Ai}}}{\sqrt{v_{\mathrm{Ae}}^{2}-v_{\mathrm{Ai}}^{2}}} n, \quad n=0,1,2, \ldots$,

for the kink modes and by

$(a / L)_{\mathrm{c}}=\frac{v_{\mathrm{Ai}}}{\sqrt{v_{\mathrm{Ae}}^{2}-v_{\mathrm{Ai}}^{2}}}\left(n+\frac{1}{2}\right), \quad n=0,1,2, \ldots$,

for the sausage modes. In these formulas $n=0$ corresponds to the fundamental mode, $n=1$ to the first harmonic, etc.

Since $\omega_{\mathrm{c}}$ is the frequency below which trapped modes are allowed, it is referred as the cut-off frequency. This frequency also appears in the problem of a pulse propagation in a uniform medium (see Rae \& Roberts 1982; Terradas et al. 2005). 

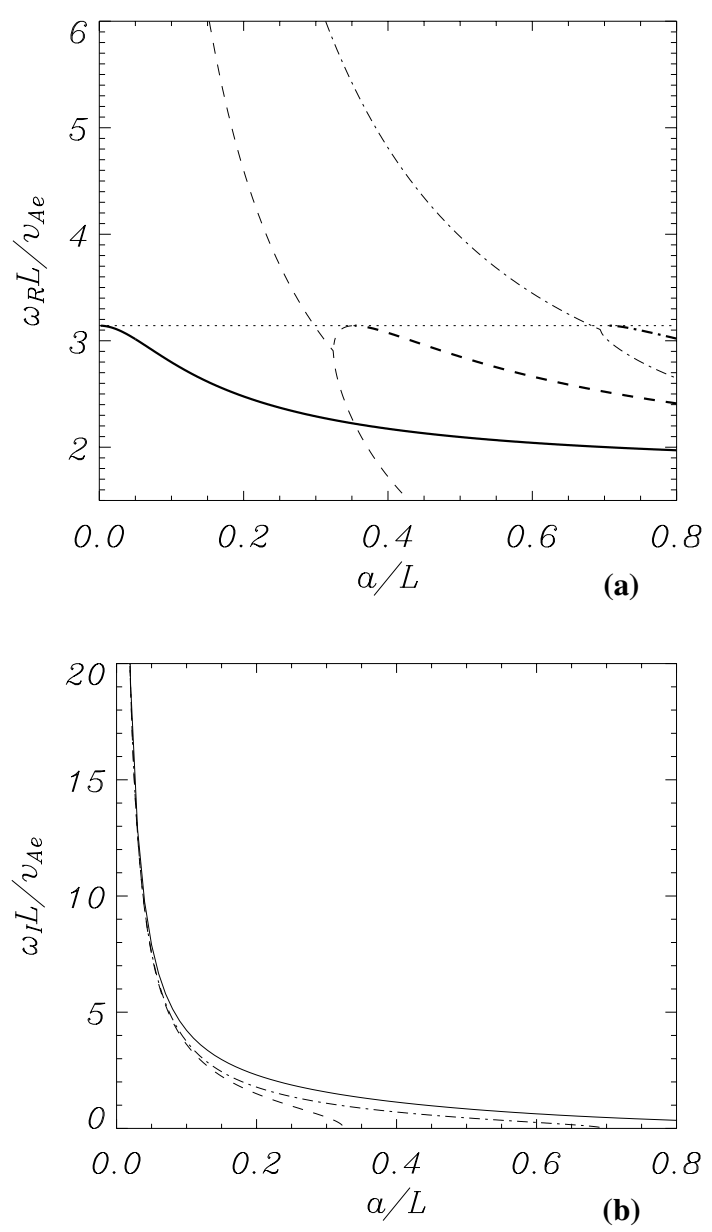

Fig. 1. Dispersion diagram calculated from the numerical solution of the dispersion relations, Eqs. (4) and (5), for a slab model with density contrast $\rho_{\mathrm{i}} / \rho_{\mathrm{e}}=3$. a) Variation of the real part of the frequency, $\omega_{\mathrm{R}}$, versus the loop radius, $a$, for the fundamental kink mode (solid line), the fundamental sausage mode (dashed line) and the first kink harmonic (dot-dashed line). The cut-off frequency, $\omega_{\mathrm{c}}$, is represented by a horizontal dotted line. Thick curves represent trapped modes while thin lines correspond to leaky modes. b) Variation of the imaginary part of the frequency, $\omega_{\mathrm{I}}$, versus the loop radius. As expected from Eqs. (11) and (13) all the modes tend to the same $\omega_{\mathrm{I}}$ for $a / L \ll 1$.

In the upper row of Fig. 2 we have plotted $v_{x}$ for the trapped modes as a function of the coordinate $x$ for $a / L=0.05$ and 0.5 (where the last number represents a thick, unrealistic loop, since typical values are $0.02<a / L<0.06$ ). The sausage mode (odd parity) has the extrema located close to the slab edge. On the other hand, the kink eigenfunction (even parity) is more localised around $x=0$ for a slab with $a / L=0.5$ than for a slab with $a / L=0.05$. The eigenfunction of trapped modes always tends to zero as $x \rightarrow \pm \infty$.

\subsection{Leaky modes}

Modes with complex frequency $\left(\omega=\omega_{R}+i \omega_{I}\right)$ are leaky and correspond to the solutions of the dispersion relation with $k_{\mathrm{e}}=-\left(k_{z}^{2}-\omega^{2} / v_{\mathrm{Ae}}^{2}\right)^{1 / 2}$; these modes are interpreted as waves radiated by (and draining energy from) the slab. Figure 1a displays the real part of the frequency, $\omega_{\mathrm{R}}$, for the fundamental
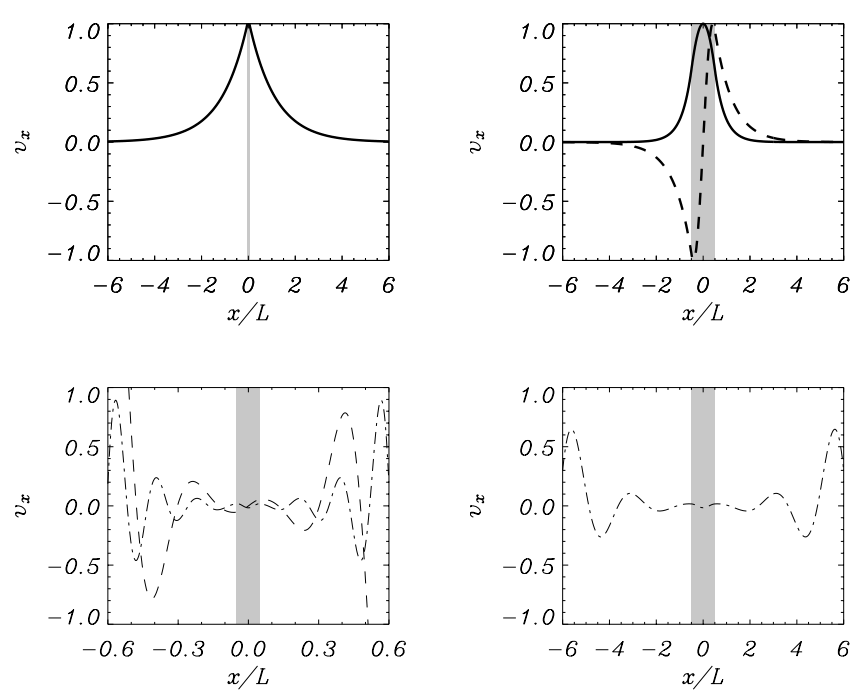

Fig. 2. Plot of the eigenfunction $v_{x}$ for the trapped modes (upper row) and some leaky modes (lower row). Line styles follow from Fig. 1: continuous line for the fundamental kink mode, dashed line for the sausage mode and dot-dashed line for the first kink harmonic. The left column corresponds to a slab with $a / L=0.05$ while for the right column $a / L=0.5$. Note that the sausage mode, that is trapped in a thick slab, becomes leaky in a thin one; also note the different spatial scale for the plot of the leaky modes with $a / L=0.05$. The grey area represents the density enhancement.

sausage mode and first kink harmonic, which are leaky for small values of the loop thickness. As $a / L$ is increased, the curves cross the cut-off frequency and bifurcate (giving rise to two branches) close to the location where the corresponding trapped mode intersects $\omega_{\mathrm{c}}$. In fact, a branch of the leaky mode connects with the trapped solution. On the other hand, the second branch has $\omega_{\mathrm{I}}=0$ and consequently is of no physical interest since its eigenfunction grows exponentially with distance from the slab ( $k_{\mathrm{e}}$ is negative and real) with a non-oscillatory spatial behaviour. In Fig. 1b we have plotted $\omega_{\text {I }}$ for the first three leaky modes. As expected, for the fundamental sausage and first kink harmonic $\omega_{\mathrm{I}}$ tends to zero as $a / L$ approaches the corresponding bifurcation point. In addition, there is a solution with a purely imaginary frequency (continuous line in Fig. 1b), which again grows with distance from the loop and so is irrelevant.

It is possible to calculate analytical approximations to the frequency of the leaky modes in the limit $a / L \ll 1$ (see Cally 1986, for the cylindrical problem). For the kink modes,

$\omega_{\mathrm{R}} \approx \frac{v_{\mathrm{Ai}}}{a} n \pi, \quad n=0,1,2, \ldots$,

$\omega_{\mathrm{I}} \approx \frac{v_{\mathrm{Ai}}}{a} \frac{1}{2} \ln \left|\frac{1+v_{\mathrm{Ai}} / v_{\mathrm{Ae}}}{1-v_{\mathrm{Ai}} / v_{\mathrm{Ae}}}\right|$,

whereas for the sausage modes,

$\omega_{\mathrm{R}} \approx \frac{v_{\mathrm{Ai}}}{a}\left(n+\frac{1}{2}\right) \pi, \quad n=0,1,2, \ldots$,
$\omega_{\mathrm{I}} \approx \frac{v_{\mathrm{Ai}}}{a} \frac{1}{2} \ln \left|\frac{1+v_{\mathrm{Ai}} / v_{\mathrm{Ae}}}{1-v_{\mathrm{Ai}} / v_{\mathrm{Ae}}}\right|$.

Equations (11) and (13) are identical and independent of $n$, so in the limit $a / L \ll 1$ all leaky harmonics (i.e. modes with 
different $n$, either kink or sausage) have the same damping time; in addition, the damping time is

$\omega_{\mathrm{I}}^{-1} \approx a v_{\mathrm{Ae}} / v_{\mathrm{Ai}}^{2}$,

for $v_{\mathrm{Ai}} \ll v_{\mathrm{Ae}}$. Similar results are found in a cylinder (see Cally 2003), although in the cylindrical loop there is another family of modes with complex frequency below the cut-off (the "principal leaky modes" following Cally's notation) that are not allowed in the slab. The leaky modes of the slab are akin to Cally's "trig" modes. An additional feature of leaky modes for $a / L \ll 1$ is that they have large frequencies, i.e. very short periods, in comparison with the frequencies of trapped modes (see Fig. 1a). Finally, the solution with $n=0$ in Eqs. (10) and (11) corresponds to the purely imaginary frequency represented with a solid line in Fig. 1b.

The value of $a / L$ in the dispersion diagram for which the leaky modes intersect the cut-off frequency is easily derived from Eqs. (10) and (12),

$(a / L)_{\mathrm{c}} \approx \frac{v_{\mathrm{Ai}}}{v_{\mathrm{Ae}}} n, \quad n=1,2,3, \ldots$,

for the kink modes, and

$(a / L)_{\mathrm{c}} \approx \frac{v_{\mathrm{Ai}}}{v_{\mathrm{Ae}}}\left(n+\frac{1}{2}\right), \quad n=0,1,2, \ldots$,

for the sausage modes. Because of the assumption $a / L \ll 1$ these equations are valid only for low order modes ( $n$ small). As expected from Fig. 1a, Eqs. (15) and (16) are different from those derived for the trapped modes (Eqs. (8) and (9)) and so for a given mode the trapped and leaky solutions touch the cutoff frequency at different values of $a$ in the dispersion diagram. Nevertheless, in the situation $v_{\mathrm{Ae}} \gg v_{\mathrm{Ai}}$ the critical values of $a / L$ for trapped modes tend to those of leaky modes.

The eigenfunctions of the fundamental leaky sausage mode $(n=0)$ and the first kink harmonic leaky mode $(n=1)$ are displayed in Fig. 2 (lower row) for $a / L=0.05, a / L=0.5$. The leaky modes are characterised by an external solution that is spatially oscillatory and grows exponentially with the distance from the slab since $k_{\mathrm{e}}$ is complex with negative real part. When higher harmonics are considered (that is, when $n$ is increased) the imaginary part of $k_{\mathrm{e}}$ also increases and as a consequence the eigenfunction has smaller scales (shorter wavelengths).

\section{Initial-value problem: temporal evolution}

Trapped modes provide with information about the stationary state of the system, but coronal oscillations are often produced by an impulsive event and a time dependent simulation is more appropriate to describe the evolution of the system. If the system relaxes to a stationary state after an initial disturbance, then it is described by the normal mode results. To study the effect of an arbitrary initial perturbation we have excited a typical loop $\left(a / L=0.05, \rho_{\mathrm{i}} / \rho_{\mathrm{e}}=3\right)$ with different velocity profiles. The perturbations are located mainly inside the slab, but for disturbances located outside the loop see Terradas et al. (2005).

To solve Eq. (3) numerically, the code PDE2D (Sewell 2002), based on finite elements, has been used. The code makes use of a second order implicit Crank-Nicholson method with adaptive time step control. Since we are considering a finite numerical domain, reflections at the domain boundaries may affect the dynamics of the slab. We have solved this problem by locating the edges of the numerical domain far from the slab. Given that the size of the domain is much larger than the loop thickness, a non-uniform grid with 1000 grid points in the full domain, 50 of them located inside the slab $(|x| \leq a)$, has been used. In addition, we have ensured that the artificial diffusion introduced by the numerical scheme is small enough. This is a critical point since artificial damping can be larger than the physical damping, in our case arising from energy leakage, and may lead to the wrong interpretation of the results. We have performed different simulations increasing the number of points and have found that the solutions converge, which is an indication that numerical diffusion does not affect considerably the results. In addition, we have checked that we get the same results by solving the initial-value problem with a standard explicit method based on finite differences.

\subsection{Sausage-like excitation}

Our first initial perturbation is odd with respect to $x=0$ and so sausage modes are excited because their eigenfunction $v_{x}$ has the same parity (see dashed line in the upper row, right column of Fig. 2). From the dispersion relation (Fig. 1a) we know that for $a / L=0.05$ there are no trapped sausage modes, while there is an infinite number of leaky sausage modes (with frequencies given by Eq. (12)). There are also trapped and leaky kink modes, but they are not excited since their parity is the opposite to that of the initial perturbation.

We consider an initial perturbation of the form

$v_{x}(x, t=0)=v_{x 0}\left\{\exp \left[-\left(\frac{x-x_{0}}{w}\right)^{2}\right]-\exp \left[-\left(\frac{x+x_{0}}{w}\right)^{2}\right]\right\}$,

where $v_{x 0}$ is the amplitude of the perturbation, $\pm x_{0}$ the position of the Gaussians centre and $w$ their width at half height. Since the numerical computations apply to the linear regime, the particular value of $v_{x 0}$ is not relevant and has been set to unity.

We have first taken $x_{0}=a$ and $w=2 a$; the results of the simulation are displayed in Fig. 3, where we have plotted $v_{x}$ at different times as a function of $x$. The initial perturbation produces travelling disturbances to the left and right and these disturbances show some dispersion as they propagate. In addition, after a short time the velocity has an almost null amplitude inside the slab. In Fig. 4 the velocity in the internal point $x=a / 2$ is plotted (note that $v_{x}$ at $x=0$ is zero because of the sausage-type perturbation). The signal at this position is of short duration, quickly attenuated and clearly showing oscillations. We have fitted this signal with a function of the form $A \cos \left(\omega_{\mathrm{R}} t+B\right) \mathrm{e}^{-t \omega_{\mathrm{I}}}$ where $\omega_{\mathrm{R}}$ and $\omega_{\mathrm{I}}$ are calculated from the corresponding analytical expressions (Eqs. (12) and (13) with $n=0)$ and $A$ and $B$ are determined by a least square fit. The fitted function is shown with a dashed line in Fig. 4 and we can see that the match with the original signal is excellent for $t>0.2 \tau_{\mathrm{A}}$. Then, it is found that the dominant period and damping time agree quite well with the period and attenuation time of the fundamental sausage leaky mode. This indicates that the fundamental sausage leaky mode has been excited and, as a 

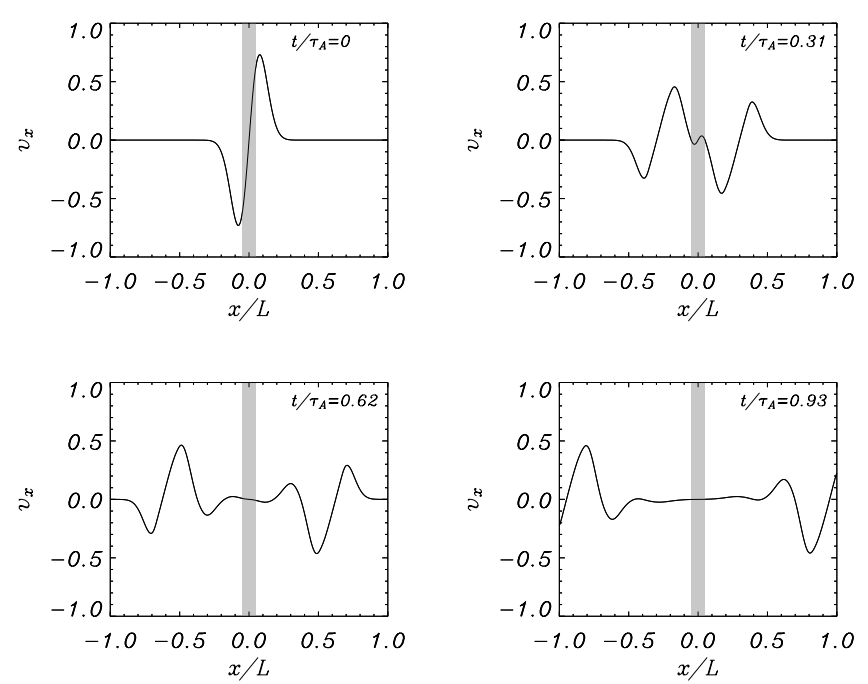

Fig. 3. Plot of $v_{x}$ for different times (in Alfvén transit time units, $\tau_{\mathrm{A}}=$ $L / v_{\mathrm{Ae}}$ ). The initial perturbation is given by Eq. (17) with $x_{0}=a, w=$ $2 a$ and $a=0.05 L$. The grey area represents the density enhancement.

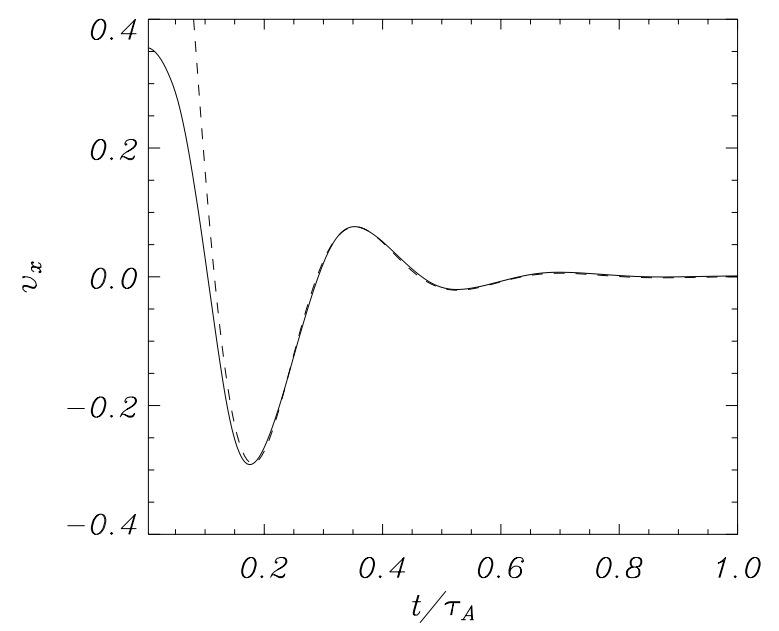

Fig. 4. Plot of $v_{x}$ as a function of time at $x=a / 2$ for the numerical simulation of Fig. 3 (continuous line). The dashed line is a fit of the form $A \cos \left(\omega_{\mathrm{R}} t+B\right) \mathrm{e}^{-t \omega_{\mathrm{I}}}$, where $\omega_{\mathrm{R}}$ and $\omega_{\mathrm{I}}$ are calculated from the corresponding analytical expressions for the fundamental leaky sausage mode (Eqs. (12) and (13) with $n=0$ ) and $A$ and $B$ are determined by a least square fit.

consequence, that the energy deposited initially in the loop is simply radiated through this leaky mode. We refer to this phase as the impulsive leaky phase.

The short interval before the slab oscillates with the leaky mode $\left(t<0.2 \tau_{\mathrm{A}}\right)$ is related to the time the perturbations need to travel inside the slab and feel the effect of its boundaries. In any case, this time interval is quite small and is of the order of the time to cross the slab width, i.e. $2 a / v_{\mathrm{Ai}} \approx 0.17 \tau_{\mathrm{A}}$.

Note that the leaky eigenfunction shows an exponential grow of the amplitude with distance from the loop (see lower row of Fig. 2). Amplitudes at large distances correspond to earlier and therefore larger amplitudes within the loop since the wave is damped with time. The leaky mode can only be stationary with this particular spatial behaviour. However, as expected, the time-dependent results do not show this grow of
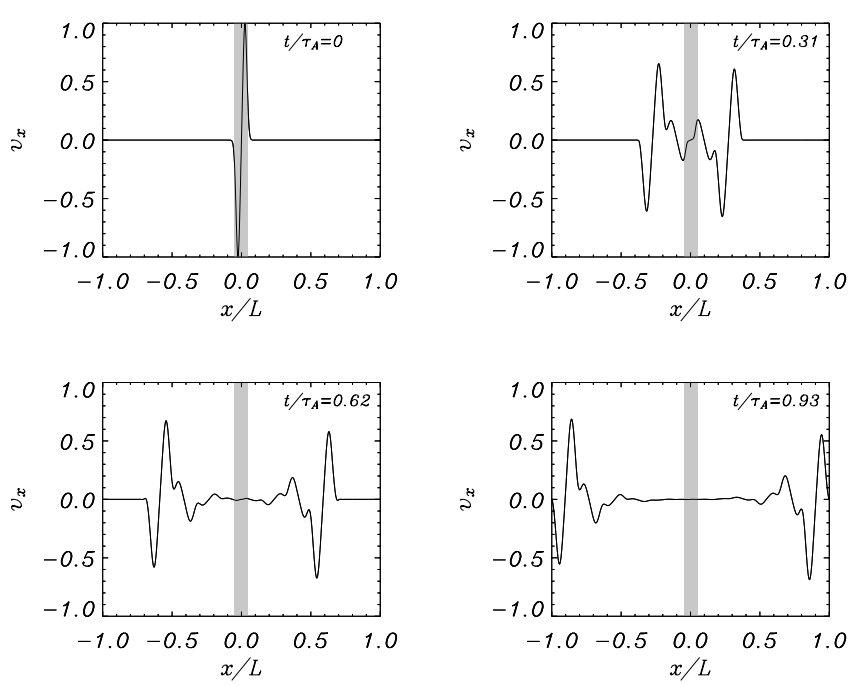

Fig. 5. Plot of $v_{x}$ for different times. The initial perturbation is given by Eq. (17) with $x_{0}=a / 2, w=2 a / 5$ and $a=0.05 L$. The grey area represents the density enhancement.

the amplitude with distance and leaky waves simply propagate dispersively away from the loop in the coronal medium.

We have next carried out a simulation for an initial perturbation given by Eq. (17) with $x_{0}=a / 2$ and $w=2 a / 5$, so this initial disturbance is more localised inside the slab (Fig. 5). The results show again the propagation of perturbations in both directions along the $x$-axis with a fast decrease of the amplitude inside the slab. Note also the presence of short scale features.

In Fig. 6a the corresponding velocity at $x=a / 2$ is plotted. Now $v_{x}$ clearly shows several periodicities and it is difficult to perform a simple fit. For this reason, we have calculated the periodogram of this signal (see Scargle 1982) to examine the dominant frequencies (Fig. 6b). This periodogram has three significant power peaks, located at the first three leaky sausage frequencies given by Eq. (12) with $n=0,1$ and 2 (dashed lines in Fig. 6b). This result indicates that three leaky sausage modes have been excited in the loop and with different strengths, since each peak has different power. The leaky mode with $n=0$ (i.e. the fundamental) is the most dominant and is the same mode that we have found in the previous simulation. The short scale features found in Fig. 5 are then due to the propagation of the first two leaky harmonics, which have shorter wavelengths in the external medium. The reason why a substantial amount of energy of the present perturbation goes into these two leaky sausage harmonics is that it contains smaller scales and so can excite solutions with shorter wavelengths.

\subsection{Kink-like excitation}

We have next excited the slab with an even perturbation of the form

$v_{x}(x, t=0)=v_{x 0} \exp \left[-\left(\frac{x}{w}\right)^{2}\right]$,

where $w=a$. The numerical computations (Fig. 7) again show that the induced disturbances propagate away from the slab and contain different wavelengths due to the dispersive nature of 

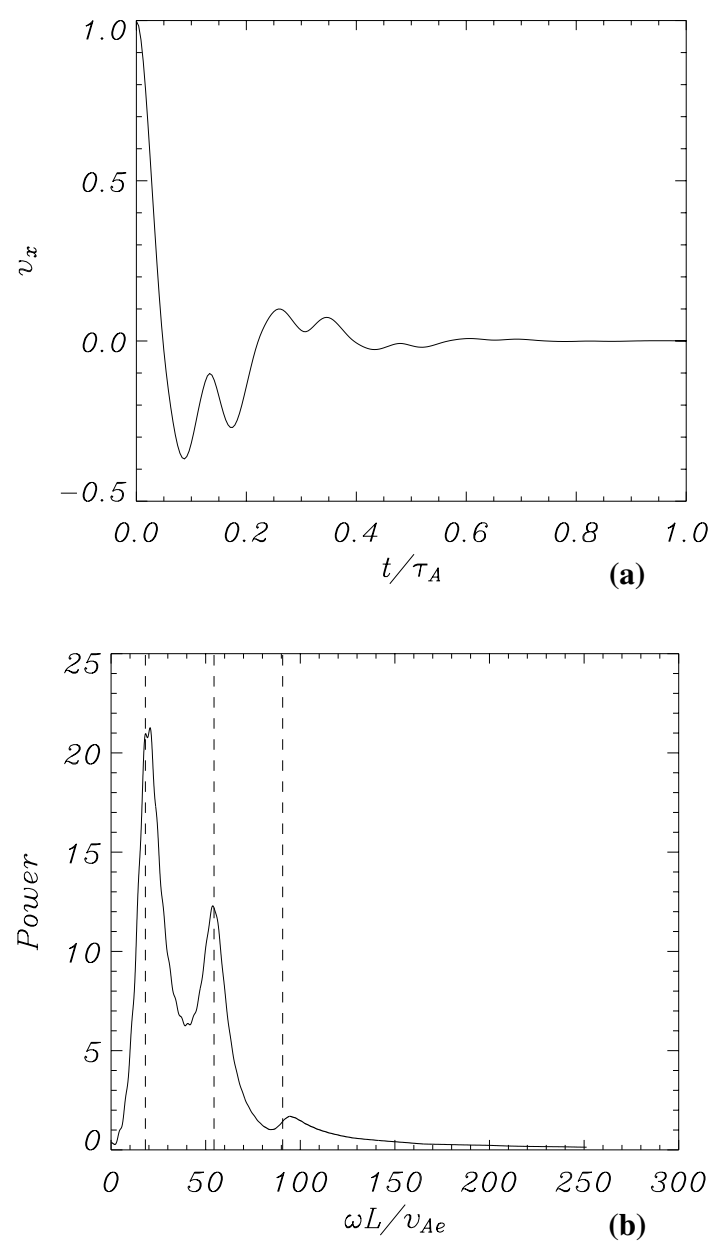

Fig. 6. a) Plot of $v_{x}$ as a function of time at $x=a / 2$ for the numerical simulation of Fig. 5. b) Periodogram of the signal shown in a). The dashed lines represent the frequencies of the first three leaky sausage modes, given by Eq. (12) with $n=0,1,2$. Since the power peaks are located at these frequencies there is a clear evidence that the first three leaky sausage modes have been excited.

fast waves. However, the most interesting feature is the shape of the velocity inside the slab and its near surroundings, that approaches the form of the fundamental kink mode eigenfunction (see upper row in Fig. 2): $v_{x}$ has an extremum at $x=0$ and decreases exponentially outside the loop. This simply indicates that the slab is oscillating with this normal mode.

In Fig. 8 we have plotted $v_{x}$ as a function of time at the slab centre $(x=0)$. We have computed a periodogram of the signal and, as expected, the frequency of oscillation for large times agrees quite well with that of the fundamental kink mode calculated by solving the dispersion relation. Note that the amplitude when the slab oscillates with the normal mode is constant but smaller than the initial amplitude $\left(v_{x 0}\right)$. Like for the sausage-like simulations, there is a short time interval before the loop reaches the stationary regime. In Fig. 8 we have plotted a detail of this transient, which is initially dominated by the propagation of the initial disturbance inside the slab $\left(0 \leq t / \tau_{\mathrm{A}}<2 a / v_{\mathrm{Ai}}\right)$ and is followed by the excitation of a leaky mode (for $2 a / v_{\mathrm{Ai}} \leq t / \tau_{\mathrm{A}}$ ) because of the reflection of the disturbance at the slab boundaries. During this impulsive leaky
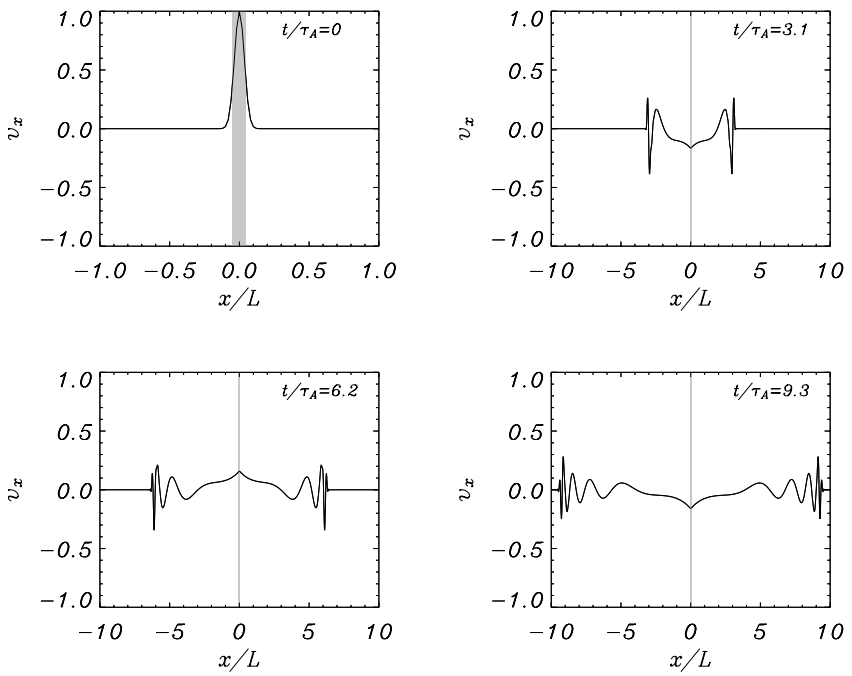

Fig. 7. Plot of $v_{x}$ for different times. The initial perturbation is given by Eq. (18) with $w=a$ and $a=0.05 L$. Note the different spatial scale for $t=0$. The grey area represents the density enhancement.

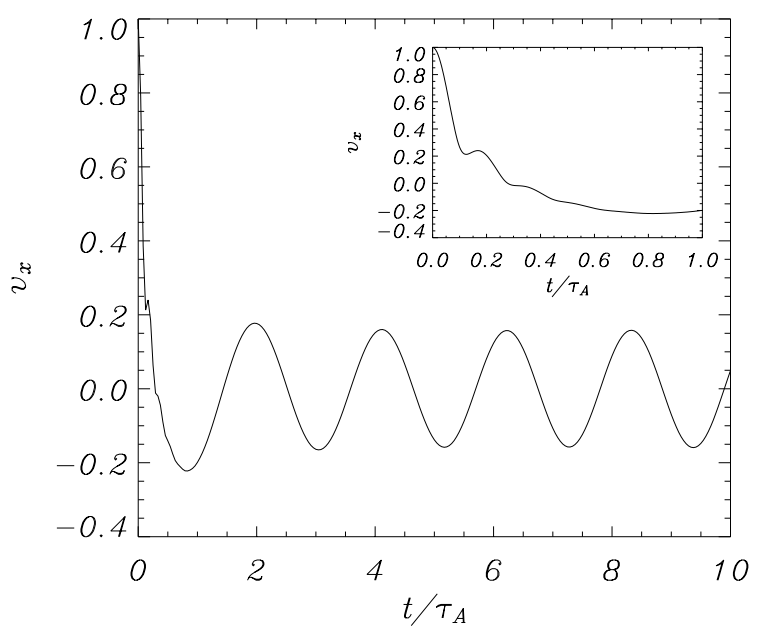

Fig. 8. Plot of $v_{x}$ at the slab centre $(x=0)$ for the numerical simulation of Fig. 7. After a short transient phase the loop reaches a stationary state, oscillating at the frequency predicted by the normal mode analysis. In the inner plot the impulsive leaky phase is displayed, showing the signal for $t / \tau_{\mathrm{A}}$ between 0 and 1 .

phase the amplitude shows short period oscillations. The period of these oscillations, which are now superimposed to the much larger period of the trapped kink mode, agrees with the period of the first leaky mode given by Eq. (10) (with $n=1$ ). Now, part of the initially deposited energy in the slab is radiated away through the fundamental kink leaky mode before the loop oscillates with the corresponding trapped mode.

Finally, it is illustrative to study the features of an initial perturbation that is more structured inside the loop,

$$
\begin{aligned}
v_{x}(x, t=0)= & v_{x 0}\left\{\exp \left[-\left(\frac{x}{w}\right)^{2}\right]-\exp \left[-\left(\frac{x-x_{0}}{w}\right)^{2}\right]\right. \\
& \left.-\exp \left[-\left(\frac{x+x_{0}}{w}\right)^{2}\right]\right\},
\end{aligned}
$$

where $x_{0}=a$ and $w=a / 2$. This perturbation has a maximum at the centre and a minimum at both edges of the slab. From the 

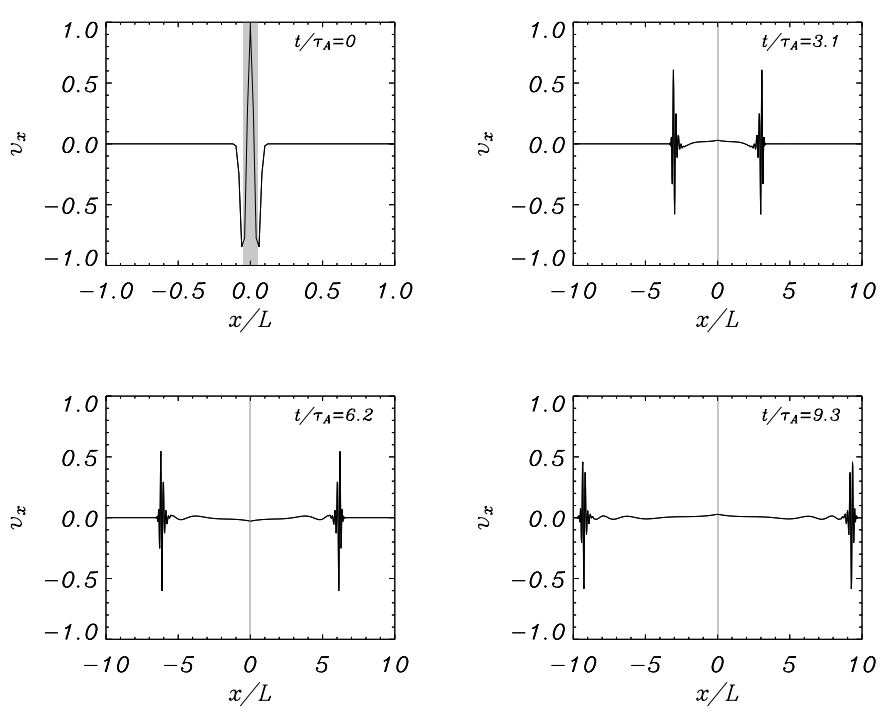

Fig. 9. Plot of $v_{x}$ for different times. The initial perturbation is given by Eq. (19) with $x_{0}=a, w=a / 2$ and $a=0.05 L$. Note the different spatial scale for the plot at $t=0$. The grey area represents the density enhancement.

temporal evolution (Fig. 9) we can see that after the transient the energy is redistributed in such a way that only the extremum at $x=0$ remains while the other extrema in the initial perturbation have disappeared: the slab is simply oscillating with the fundamental kink mode.

In Fig. 10 we see that the velocity at $x=0$ shows an impulsive leaky phase (plotted in more detail in the inset) with a very large amplitude in comparison with the amplitude of the trapped fundamental kink mode. The periodogram analysis reveals that the dominant period in the impulsive leaky phase is equal to that of the first leaky mode (Eq. (10) with $n=1$ ). A comparison of Figs. 10 and 8 indicates that the amount of kinetic energy deposited in the loop, which is proportional to the square of the normal mode velocity amplitude, strongly depends on the form of the initial perturbation. Once more it is found that an initial perturbation with smaller spatial scales gives rise to the excitation of higher harmonics because of their inherent shorter wavelength.

\section{Discussion and conclusions}

The time-dependent behaviour of a line-tied slab model under different kinds of internal excitations has been studied. The simplicity of the model has allowed us to establish a direct comparison between the results of the time-dependent solutions and the normal mode information.

The normal mode analysis shows the existence of leaky and trapped modes. Leaky modes are characterised by short period oscillations and are quickly attenuated with time. Then, when leaky modes are excited their signature is a short oscillatory transient, what we call the impulsive leaky phase. On the other hand, trapped modes have in general much longer periods and represent the stationary state of the system. The dispersion diagram indicates, depending on the value of $a / L$, which leaky and trapped modes are allowed. Thus, trapped and/or leaky modes are excited according to the ratio $a / L$, but also according

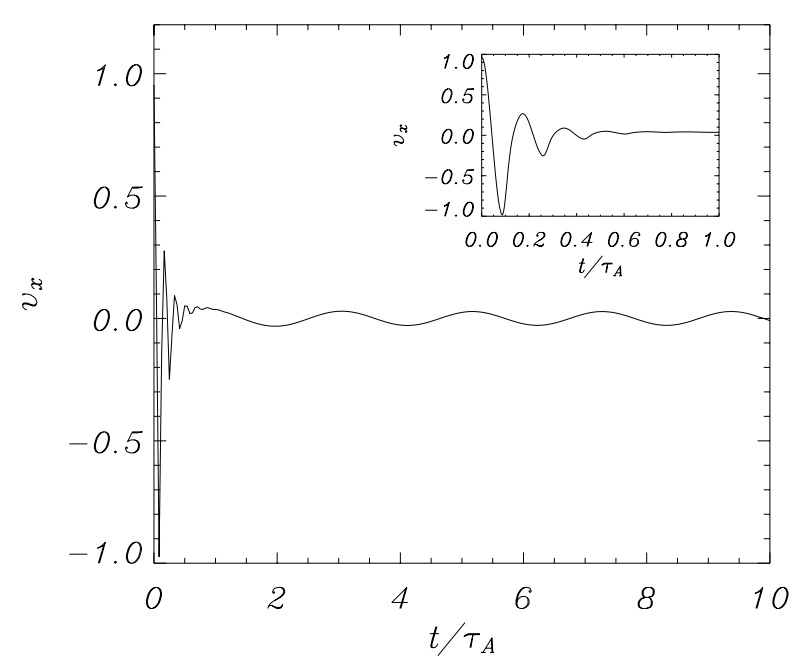

Fig. 10. Plot of $v_{x}$ at the centre of the slab $(x=0)$ for the numerical simulation of Fig. 9. After a short transient phase the loop reaches to a stationary state, oscillating at the frequency predicted by the normal mode analysis. In the inner plot the impulsive leaky phase is displayed, showing the signal for $t / \tau_{\mathrm{A}}$ between 0 and 1 .

to the shape of the initial perturbation. Firstly, initial disturbances with odd parity with respect to the loop centre can excite sausage modes, whereas even disturbances lead to the excitation of kink modes. Secondly, the size of the spatial scales in the initial perturbation determine how much energy, if any, goes into each candidate mode that can come into play, in such a way that the smaller the scales in the initial perturbation, the more energy goes into higher the harmonics. Although this issue has not been quantified, an important conclusion that must be learnt is that one can get a qualitative description of the time evolution of the slab from the information in the dispersion diagram.

We have studied the temporal evolution of sausage- and kink-type excitations for a typical coronal loop with $a / L=0.05$ and $\rho_{\mathrm{i}} / \rho_{\mathrm{e}}=3$. For $0 \leq t \lesssim 2 a / v_{\mathrm{Ai}}$ there is a short transient in which perturbations adjust as they travel inside the slab and feel the presence of its boundaries. Next, if any leaky modes are excited there is an impulsive leaky phase, characterised by damped oscillations that radiate energy from the slab away into the coronal medium. Finally, all that remains is the undamped trapped mode, with some energy confined to the slab. Nevertheless, for a realistic slab thickness (such as the value $a / L=0.05$ used here) there are no trapped sausage modes, so a sausage-like initial disturbance can only deposit energy in the leaky sausage modes and the stationary phase is absent. The excitation of the fundamental sausage trapped mode is only possible for $a / L$ lager than the first sausage critical value $(a / L)_{\mathrm{c}}$ in Eq. (9), i.e. for very wide slabs. Such as expected, the period and damping time of the leaky transient and the period of the stationary phase agree with the normal mode results for fast leaky and fast trapped modes, respectively.

Regarding the oscillations of the external medium, the trapped mode is a standing wave and the plasma close to the slab oscillates with the corresponding eigenfrequency. However, at a fixed position far from the slab, and since the 
amplitude of the trapped mode decreases exponentially with distance, oscillations are not related with the normal mode. The velocity profile far from the loop is basically the same as that produced by a pulse located around $x=0$ in a uniform medium and it is possible to identify a wavefront propagating at the Alfvén speed and a wake behind this pulse oscillating at the cut-off frequency.

Because of their small period and short damping time leaky modes are very difficult to detect unless high cadence observations are available. Hence, the strong damping seen in observations by TRACE cannot be explained with the leakage of these modes, since the oscillating loops have a much longer period of oscillation. However, the leaky modes can play a relevant role in the dynamics of the loop and are very significant from the energy point of view.

Although we have restricted our analysis to a zero-beta plasma, small plasma beta would not change much the results since fast modes are only slightly modified by the pressure effect (at least for $\beta \ll 1$, which is valid for coronal conditions). The main difference when pressure is incorporated to the model is that slow modes can also be excited. Since we are mostly interested on transversal oscillations we have not considered slow modes which produce longitudinal oscillations along the slab.

The different phases of impulsively generated waves propagating along the slab, studied by Roberts et al. (1984); Murawski \& Roberts (1993a,c); Selwa \& Murawski (2004b), are not present in our study since in our model we have made a Fourier analysis along the slab. Nevertheless, in a real excitation there is propagation along and across the loop and as a consequence the two-dimensional problem with line-tying needs to be studied.

Acknowledgements. J. Terradas thanks the Spanish Ministry of Education and Science for the funding provided under a Juan de la Cierva fellowship. The authors thank the Spanish Ministry of Science and Technology for the funding provided under grant
AYA2003-00123. Finally, the authors also thank Marc Carbonell for his help in the analytical part of the work.

\section{References}

Aschwanden, M. J., Fletcher, L., Schrijver, C. J., \& Alexander, D. 1999, ApJ, 520, 880

Aschwanden, M. J., De Pontieu, B., Schrijver, C. J., \& Title, A. M. 2002, Sol. Phys., 206, 99

Aschwanden, M. J., Nightingale, R. W., Andries, J., Goossens, M., \& Van Doorsselaere, T. 2003, ApJ, 598, 1375

Cally, P. S. 1986, Sol. Phys., 103, 277

Cally, P. S. 2003, Sol. Phys., 217, 95

Díaz, A. J., Oliver, R., Ballester, J. L., \& Roberts, B. 2004, A\&A, 424, 1055

Edwin, P. M., \& Roberts, B. 1982, Sol. Phys., 79, 239

Edwin, P. M., \& Roberts, B. 1983, Sol. Phys., 88, 179

Huang, P. 1996, Phys. Plasmas, 3, 2579

Huang, P., Musielak, Z. E., \& Ulmschneider, P. 1999, A\&A, 342, 300

Musielak, Z. E., Huang, P., \& Ulmschneider, P. 2000, 362, 359

Murawski, K., \& Roberts, B. 1993a, Sol. Phys., 143, 89

Murawski, K., \& Roberts, B. 1993b, Sol. Phys., 144, 101

Murawski, K., \& Roberts, B. 1993c, Sol. Phys., 144, 255

Nakariakov, V. M., \& Ofman, L. 2001, A\&A, 372, L53

Nakariakov, V. M., Ofman, L., DeLuca, E. E., Roberts, B., \& Davila, J. M. 1999, Science, 285, 862

Rae, I. C., \& Roberts, B. 1982, ApJ, 256, 761

Roberts, B, Edwin, P. M., \& Benz, A. O. 1984, ApJ, 279, 857

Scargle, J. D. 1982, ApJ, 263, 835

Schrijver, C. J., Aschwanden, M. J., \& Title, A. M. 2002, Sol. Phys., 206, 69

Selwa, M., Murawski, K. 2004a, A\&A, 422, 1067

Selwa, M., Murawski, K. 2004b, A\&A, 425, 719

Sewell, G. 2002, Finite Differences, Finite Elements and PDE2D

Spruit, H. C. 1982, Sol. Phys., 75, 3

Terradas, J., \& Ofman, L. 2004, ApJ, 610, 523

Terradas, J., Oliver, R., \& Ballester, J. L. 2005, ApJ, 618, L149

Uralov, A. M. 2003, Astron. Lett., 29, 486 